\title{
Teoria de Orem aplicada a intervenciones durante embarazo y climaterio
}

\author{
Aplicação da Teoria de Orem em intervenções durante a gestação e climatério \\ Applications of Orem's Theory in addressing interventions during pregnancy and climateric
}

\author{
Alide Salazar Molina', Sandra Valenzuela Suazo' \\ 'Universidad de Concepción. Departamento de Enfermería. Programa de Doctorado en Enfermería. Concepción, Chile
}

Submissão: $13 / 08 / 2008$

Aprovação: $20 / 07 / 2009$

\section{RESUMO}

El objetivo de este trabajo es desarrollar aplicaciones de la teoría de Autocuidado de Orem, para el abordaje de intervenciones de enfermería el embarazo y climaterio, y determinar su utilidad como un marco teórico para el abordaje del autocuidado durante las fases de desarrollo. Se concluye que la aplicación de este marco teórico, en la asistencia de mujeres durante el embarazo y climaterio, por parte de enfermeras y enfermeras obstétricas contribuye a identificar los requisitos de autocuidado, las intervenciones requeridas, la necesidad de autocuidado y los métodos de ayuda Que las mujeres requieren con especial énfasis en la capacitación y desarrollo de la capacidad de autocuidado.

Descriptores: Autocuidado; Teoría de enfermería; Embarazo; Climaterio.

\section{RESUMO}

O objetivo deste trabalho foi desenvolver aplicações da Teoria do Autocuidado de Orem para realizar intervenções em enfermagem durante a gestação e climatério, mas tentamos também determinar a sua utilidade como marco teórico para pensar (ou) planejar o autocuidado nestas fases da vida da mulher. Pudemos observar Que a aplicação desta teoria e a sua utilidade como marco teórico na assistência à mulheres durante a gestação e climatério, por parte de enfermeiras e enfermeiras obstétricas, contribuiu não só para identificar os ręuisitos necessários para o autocuidado e planejar as intervenções ręueridas e as necessidades específicas de autocuidado, mas constatamos também que esta pode ser a base para elaboração de métodos de desenvolvimento da ampliação da capacidade feminina para o auto-cuidado.

Descitores: Autocuidado; Teoria de Enfermagem; Gravidez; Climatério.

\section{ABSTRACT}

The aim of this paper is to develop applications of Orem's Theory of Self Care in order to address nursing interventions during pregnancy and menopause, and to determine its usefulness as a theoretical framework for addressing self care during stages of development. It is concluded that the application by nurses and midwives of this theoretical framework, in assisting women during pregnancy and menopause, contributes to identifying the requirements of self care, the required interventions, necessity of self care and the methods of assistance that women require with special emphasis on training and development of self care procedures.

Descriptors: Selfcare; Nursing Theory; Pregnancy; Climacteric. 


\section{INTRODUCCIÓN}

Desde la década pasada, algunos investigadores han utilizado la teoría del déficit del autocuidado de Orem, para el abordaje de los más diversos pacientes, en vista Que facilita el planeamiento de la asistencia y se ha mostrado efectiva y eficaz para hacer que los individuos se ocupen de su autocuidado(I). Es así como se han publicado algunos trabajos Que abordan el estudio de las mujeres durante el embarazo ${ }^{(1-3)}$ y durante la fase de climaterio ${ }^{(4-6)}$.

Respecto de la aplicación de la teoría para el abordaje de mujeres durante la fase de embarazo, el foco de la investigación ha estado centrado fundamentalmente en la identificación de requisitos universales y de desviación de la salud en situaciones de alto riesgo obstétrico. Esta situación ha sido algo distinta para las investigaciones Que han abordado la aplicación de esta teoría durante la etapa de climaterio, sin embargo el foco ha estado centrado en los requisitos universales más Que en los del desarrollo.

Desde la perspectiva de los cuidados preventivos de la salud, satisfacer de manera efectiva los requisitos de autocuidado universales y de desarrollo en los individuos sanos está en la naturaleza de la prevención primaria de la enfermedad y de las alteraciones de la salud ${ }^{(7)}$. De ahí la necesidad de desarrollar aplicaciones de la teoría de Autocuidado de Dorotea Orem dirigidas al abordaje de los requisitos del desarrollo específicos de los períodos de embarazo y climaterio, y de este modo contribuir a la promoción de la salud y prevención de complicaciones derivadas de estas fases de desarrollo en la mujer.

\section{OBJETIVOS}

- Identificar requisitos de autocuidado relativos a la etapa de embarazo fisiológico y climaterio e identificar posibles déficit de autocuidado.

- Detectar posibles factores que obstaculicen la realización del autocuidado.

- Identificar posibles déficit de autocuidado.

- Desarrollar planificación del cuidado de enfermería.

- Determinar la utilidad de la teoría de Autocuidado de Dorotea Orem, como marco teórico para el abordaje del cuidado y autocuidado durante las fases de desarrollo señaladas.

\section{TEORÍA GENERAL DEL DÉFICIT DEL AUTOCUIDADO}

La Teoría general del déficit del autocuidado planteada por Orem en $1980^{(7)}$, ha sido extensamente utilizada en la investigación sobre autocuidado. Orem ${ }^{(8)}$ etieueta su teoría como una teoría general compuesta por las siguientes tres subteorías relacionadas:

a) La teoría del déficit del autocuidado, Que describe y explica cómo la enfermería puede ayudar a la gente.

b) La teoría de autocuidado, Que describe el poreué y el cómo las personas cuidan de sí mismas.

c) La teoría de sistemas enfermeros, Que describe y explica las relaciones Que hay Que mantener para que se produzca el cuidado enfermero.

\section{Autocuidado}

El autocuidado consiste en la práctica de las actividades Que las personas maduras, o que están madurando, inician y llevan a cabo en determinados períodos de tiempo, por su propia parte y con el interés de mantener un funcionamiento vivo y sano, y continuar con el desarrollo personal y el bienestar mediante la satisfacción de requisitos para las regulaciones funcional y del desarrollo( ${ }^{(8)}$.

En el término autocuidado, la palabra "auto" se utiliza en el sentido del ser humano completo. El autocuidado comporta la connotación dual de "para uno mismo" y "realizado por uno mismo". El autocuidado tiene un propósito. Es una acción que tiene un patrón y una secuencia y, cuando se realiza efectivamente, contribuye de manera específica a la integridad estructural, desarrollo $y$ funcionamiento humanos ${ }^{(7)}$.

\section{Requisitos de Autocuidado}

Un reQuisito de autocuidado es un consejo formulado y expresado sobre las acciones Que se deben llevar a cabo poreue se sabe o se supone Que son necesarias para la regulación de los aspectos del funcionamiento y desarrollo humano, ya sea de manera continua o bajo unas circunstancias y condiciones específicas.

Los requisitos de autocuidado formulados y expresados representan los objetos formalizados del autocuidado. Son las razones por las Que se emplea el autocuidado; expresan los resultados deseados ${ }^{(8)}$. Tres son los requisitos que se han identificado en esta teoría:

- Requisitos universales: están asociados con el proceso de la vida o integran el funcionamiento humano. Son comunes a todos los seres humanos.

- Requisitos del desarrollo. Están asociados con el proceso y condiciones específicas a los estados de desarrollo de la persona. Se definen tres conjuntos de requisitos de autocuidado del desarrollo: a) Provisión de condiciones Que fomentan el desarrollo, b) Implicación en el autodesarrollo, c) Prevenir o vencer efectos de las condiciones y de las situaciones vitales Que pueden afectar de manera negativa el desarrollo humano. Los requisitos de autocuidado del desarrollo son expresiones especializadas de los requisitos de autocuidado universal que han sido particularizadas para los procesos de desarrollo, o son nuevos requisitos derivados de una condición (ejemplo: embarazo, climaterio), o asociados con un acontecimiento (ejemplo: pérdida del cónyuge o pérdida de trabajo).

- Requisitos de desviación de la salud: están asociados con la regulación de los efectos y resultados de las funciones perturbadas (con la injuria y sus efectos) y con el efecto y resultados del cuidado de salud prescrito por el médico.

Estos tres tipos de requisitos deben ser especificados para los individuos y deben estar en relación a la edad, sexo, estados del desarrollo, estados funcionales, estados de vida y condiciones ambientales.

Los requisitos de autocuidado deben conocerse antes de Que puedan servir como propósitos de autocuidado. Los requisitos de autocuidado universal deberían ser conocidos por todos los adultos capaces de ser enseñados. Idealmente, lo mismo podría aplicarse a los reeuisitos de autocuidado del desarrollo. Sin embargo, no siempre se selecciona efectivamente y se organiza adecuadamente el conocimiento veraz para su divulgación pública. Los requisitos de autocuidado de desviación de la salud generalmente los llegan a conocer aquellos que tienen defectos genéticos o constitucionales o desviaciones de la salud, o miembros de la familia o amigos con 
tales defectos o desviaciones de la salud ${ }^{(7)}$

Orem $^{(7)}$ sostiene Que las enfermeras desarrollan capacidades para: identificar los requisitos de autocuidado de sus pacientes, seleccionar o confirmar los métodos generales y operaciones o acciones requeridas a través de los cuales cada requisito identificado puede y deberá ser cubierto, e identificar las acciones a realizar para satisfacer cada requisito de autocuidado específico.

\section{APLICACIÓN DE LA TEORÍA DE OREM EN EL ABORDAJE DE LOS REQUISITOS DEL DESARROLLO EN LOS PERÍODOS DE EMBARAZO Y CLIMATERIO}

Durante el embarazo la mujer experimenta diversas modificaciones, ocasionando necesidades de autocuidado y, en ocasiones déficit de autocuidado, Que deben ser resueltos o compensados para la mantención de la salud y preservación del bienestar. Para ello las embarazadas deben ser motivadas y educadas para realizar acciones de autocuidado, debiendo comprender la naturaleza de la asistencia y los factores Que intervienen en la misma ${ }^{(1)}$.

Análogamente, durante el climaterio la mujer experimenta variados síntomas/manifestaciones derivadas del declinamiento de la función ovárica y posterior cese de la función folicular ${ }^{(10)}$ Que provocan necesidades de autocuidado y algún grado de déficit ${ }^{(11-13)}$ Que deben ser atendidos o compensados.

En ambas etapas, los requisitos de autocuidado del desarrollo estarían definidos por cambios físicos, psicológicos, y sociales, por lo Que las acciones de autocuidado desarrolladas estarán destinadas a promover y mantener la salud, y a mejorar o aliviar los efectos negativos a causa de los cambios experimentados durante estos períodos.

Continuando con el esquema propuesto por Orem ${ }^{(7)}$ :

- Se identificaran, formularan y expresaran los requisitos de autocuidado del desarrollo característicos de los períodos de embarazo y climaterio

- Posteriormente se determinarán los métodos o tecnologías (validos y fiables) para satisfacer el requisito en las condiciones y circunstancias humanas y ambientales predominantes.

- Se establecerán grupos y secuencias de acciones como medio a través del cual se satisfará el requisito concreto.

- Se identificará la presencia de condiciones humanas y ambientales Que: a) permiten satisfacer el requisito; o b) no lo permiten y constituyen obstáculos o interferencias para ello (ejm. edad, orientación sociocultural, disponibilidad de recursos)

- Se identificaran los posibles déficit de autocuidado en la satisfacción de las necesidades actuales o proyectadas de autocuidado terapéutico y serán definidos los papeles Que el paciente o la/el enfermera/o desarrollaran para Que las exigencias de autocuidado sean atendidas.

- Se elaborará la planificación de enfermería de acuerdo los posibles déficit identificados, identificando si se trata de un sistema de enfermería totalmente compensatorio, parcialmente compensatorio o de apoyo educativo

Las tablas 1, 2, 3 y 4 (apendice 1) muestran ejemplos de sistemas de acción para satisfacer requisitos de autocuidado del desarrollo particularizados para el embarazo y climaterio y la planificación de enfermería de acuerdo los posibles déficits para ambos períodos.

\section{CONSIDERACIONES FINALES}

Durante el embarazo y climaterio, las mujeres y sus familias emprenden una serie de actividades tradicionales y no-tradicionales destinadas a mantener la salud, y a mejorar o aliviar los efectos negativos a causa de los cambios Que experimentan durante estos período.

La aplicación del modelo de Orem, permite la identificación de los requisitos específicos de esta etapa, los grupos de operaciones requeridas y la necesidad de autocuidado que contribuye a establecer un punto de partida para la aplicación del proceso de enfermería, con especial énfasis en la capacitación y desarrollo de la capacidad de autocuidado de las propias mujeres y sus familias.

La mezcla de requisitos de autocuidado en la necesidad de autocuidado terapéutico y la complejidad de la necesidad de cuidados continuados de los individuos es una muestra de los tipos de conocimiento y la diversidad de habilidades requeridas por las personas y los profesionales de enfermería obstétrica Que actúan para satisfacer las demandas.

Cabe mencionar Que los requisitos de autocuidado del desarrollo identificados en este trabajo no constituyen una lista exhaustiva, sino más bien una aproximación, Que facilite el abordaje del proceso de enfermería de particular utilidad para a formación académica de los estudiantes en los distintos niveles de la disciplina.

Se concluye Que a aplicación de este marco teórico para la implementación del proceso de enfermería puede mejorar la atención proporcionada a las mujeres en las fases de desarrollo abordadas, así como durante otras fases del crecimiento y desarrollo.

\section{REFERÊNCIAS}

1. Farias MC, Nóbrega MM. Diagnósticos de enfermagem numa gestante de alto risco baseados na teoria do autocuidado de Orem: estudo de caso. Rev Latino-am Enfermagem 2000; 8 (6): 59-67.

2. Hart M, Foster S. Self-Care Agency in two groups of pregnant women. Nurs Sci Quarterly 1998; I I (4): 167-7I.

3. Torres GV, Davim RM, Nóbrega M.M. Aplicação do processo de enfermagem baseado na teoria de orem: estudo de caso com uma adolescente grávida. Rev Latino-am Enfermagem 1999; 7 (2): 47-53.

4. Mamede M, Nakano A, Felipe M, Messias D. Respostas a auto cuidado de mulheres brasileiras em perimenopausa: estudo piloto. Rev Bras Enferm 1991; 44 (2/3): 26-30.

5. Hartweg D. Self-Care actions of healthy middle-aged women to promote well-being. Nurs Res 1993; 42: 221-7.

6. Bernhard, L. Self-care strategies on menopause women. I Women \& Aging 1997; 9: 77-89.

7. Orem D. Modelo de Orem. Conceptos de Enfermería en la práctica. Barcelona: Ediciones Científicas y Técnicas MassonSalvat; 1993.

8. Marriner A, Raile M. Modelos y teorías en enfermería. $6^{\text {th }}$ ed. Madrid: Elsevier España S. A; 2007.

9. Pérez, I. Aplicación de las Teorías de Orem y Peplau en el Proceso de Atención de Enfermería. Congreso de Neurocirugía, 
La Habana, Cuba, 2002 [serial on the Internet]. 2002 [cited 2007 Nov 26]; Disponible en: http://www.uninet.edu/ neuroc2002/papers/TL-OREMYPEPLAU.htm

10. Arteaga E, Bianchi M. Fisiología del Climaterio y Menopausia. En: Pérez-Sánchez A, editor. Ginecología Pérez-Sánchez. Santiago: Mediterráneo; 2003.

11. Bastías E, Sanhueza O. Conductas de autocuidado y manifestaciones perimenopáusicas en mujeres de la comuna de Concepción, Chile. Ciencia Enferm 2004; 10: 41-56.

12. Salazar A, Paravic T. Desempeño laboral y climaterio en trabajadoras de instituciones públicas. Rev Med Chile. 2005; 133: 315-22.

13. Obermeyer C, Reher D, Cortes L, Price K. The menopause in Spain: results of the DAMES (Decisions At Menopause) Study. Maturitas 2005; 52: 190-8.

14. Uauy R, Atalah E, Barrera C, Behnke E. Alimentación y nutrición durante el embarazo. En: Guías de alimentación para la mujer. Santiago; 2001.

15. Donoso E. Nutrición materna y embarazo. En: Badía II ed. HTLM. Alto riesgo obstétrico. [book on the Intrenet]. Santiago, Chile: Publicaciones Universitarias Pontificia Universidad Católica de Chile; 1997 [cited 2007 Ago 10]. Disponible en:http:// escuela.med.puc.cl/paginas/Departamentos/Obstetricia/ AltoRiesgo/nutricion.html

16. Campusano C. Conceptos y definiciones en climaterio $y$ menopausia. En: Arteaga E, P Contreras P, González O. editores. Consenso en Climaterio 2001. Santiago: Sociedad Chilena de Climaterio; 2001.

17. Villaseca P, Craig IM, Pinochet M. Selección, evaluación y seguimiento de la paciente de la peri y postmenopáusia. En: Arteaga E, P Contreras P, González O. editores. Consenso en Climaterio 200 I. Santiago: Sociedad Chilena de Climaterio; 200 I.

\section{APENDICE}

\begin{tabular}{|c|c|c|c|c|c|}
\hline $\begin{array}{l}\text { Déficit de } \\
\text { Autocuidado }\end{array}$ & $\begin{array}{l}\text { Diagnóstico de } \\
\text { enfermería }\end{array}$ & Objetivo & Método de ayuda & Tipo de sistema & Intervenciones de Enfermería \\
\hline $\begin{array}{l}\text { Ingestión de } \\
\text { lácteos inferior a } \\
1.000 \mathrm{mg} / 24 \\
\text { horas }\end{array}$ & $\begin{array}{l}\text { Alteración de la } \\
\text { nutrición: } \\
\text { consumo inferior } \\
\text { de los } \\
\text { reQuerimientos } \\
\text { de lácteos }\end{array}$ & $\begin{array}{l}\text { Que la } \\
\text { embarazada } \\
\text { aumente la } \\
\text { ingesta de } \\
\text { lácteos }\end{array}$ & Educación & Apoyo educativo & $\begin{array}{l}\text {-Proporcionar instrucción acerca de necesidades } \\
\text { de nutrientes durante la gestación } \\
\text {-Proporcionar instrucción acerca de: elección de } \\
\text { alimentos y manera de prepararlos, disponibilidad } \\
\text { de costearlos. } \\
\text {-Referencia a profesional si alteración persiste. }\end{array}$ \\
\hline $\begin{array}{l}\text {-Deficiente uso } \\
\text { de técnicas de } \\
\text { relajación y } \\
\text { fortalecimiento } \\
\text { de la } \\
\text { musculatura de } \\
\text { piso pelviano y } \\
\text { abdomen para el } \\
\text { parto }\end{array}$ & $\begin{array}{l}\text { Déficit de } \\
\text { conocimientos } \\
\text { sobre técnicas de } \\
\text { relajación y } \\
\text { respiración } \\
\text { durante el parto }\end{array}$ & $\begin{array}{l}\text { Que la } \\
\text { embarazada } \\
\text { realice ejercicios } \\
\text { de respiración y } \\
\text { fortalecimiento } \\
\text { de la } \\
\text { musculatura del } \\
\text { piso pelviano y } \\
\text { abdomen para el } \\
\text { parto }\end{array}$ & Educación & Apoyo educativo & $\begin{array}{l}\text {-Proporcionar instrucción acerca de } \\
\text { modificaciones anatomo-fisiológicas Que se } \\
\text { producen durante la gestación } \\
\text {-Indicación de ejercicios Que fortalezcan } \\
\text { musculatura del piso pelviano y abdominal } \\
\text { - Referencia a curso de preparación para el parto }\end{array}$ \\
\hline $\begin{array}{l}\text { Proceso de } \\
\text { gestación no } \\
\text { monitorizado }\end{array}$ & $\begin{array}{l}\text { Mantenimiento } \\
\text { de la salud } \\
\text { alterado }\end{array}$ & $\begin{array}{l}\text { Que la } \\
\text { embarazada } \\
\text { mantenga un } \\
\text { monitoreo } \\
\text { periódico del } \\
\text { proceso de } \\
\text { gestación }\end{array}$ & $\begin{array}{l}\text { Educación } \\
\text { Guiar y dirigir }\end{array}$ & $\begin{array}{l}\text { Apoyo educativo } \\
\text { Parcialmente } \\
\text { compensatorio }\end{array}$ & $\begin{array}{l}\text {-Proporcionar instrucción acerca de objetivos del } \\
\text { control prenatal } \\
\text {-Realizar control prenatal } \\
\text {-Referencia a control con otros profesionales } \\
\text { - Referencia a curso de preparación para el parto }\end{array}$ \\
\hline $\begin{array}{l}\text { Aporte } \\
\text { profiláctico } \\
\text { inferior a } 60-100 \\
\text { mg de hierro }\end{array}$ & $\begin{array}{l}\text { Alteración de la } \\
\text { nutrición: } \\
\text { consumo inferior } \\
\text { de los } \\
\text { reQuerimientos } \\
\text { de hierro }\end{array}$ & $\begin{array}{l}\text { Que la } \\
\text { embarazada } \\
\text { adopte medidas } \\
\text { para favorecer la } \\
\text { ingesta de hierro }\end{array}$ & Educación & Apoyo educativo & $\begin{array}{l}\text {-Proporcionar instrucción acerca de necesidades } \\
\text { de nutrientes durante la gestación } \\
\text {-Proporcionar instrucción acerca de: medidas } \\
\text { Que favorezcan la absorción de hierro y } \\
\text { disponibilidad de costearlos. }\end{array}$ \\
\hline Constipación & $\begin{array}{l}\text { Alteración de la } \\
\text { eliminación } \\
\text { intestinal: } \\
\text { constipación } \\
\text { relacionado a } \\
\text { escaso consumo } \\
\text { de alimentos } \\
\text { ricos en fibra }\end{array}$ & $\begin{array}{l}\text { Que la } \\
\text { embarazada } \\
\text { adopte medidas } \\
\text { para facilitar el } \\
\text { tránsito intestinal }\end{array}$ & $\begin{array}{l}\text { Educación } \\
\text { Guiar y dirigir }\end{array}$ & $\begin{array}{l}\text { Apoyo educativo } \\
\text { Parcialmente } \\
\text { compensatorio }\end{array}$ & $\begin{array}{l}\text {-Proporcionar instrucción acerca de } \\
\text { modificaciones del tracto digestivo durante el } \\
\text { embarazo } \\
\text {-Indicación de ingesta de alimentos ricos en fibra } \\
\text {-Referencia a profesional si alteración persiste }\end{array}$ \\
\hline $\begin{array}{l}\text { Ineficaz } \\
\text { adaptación } \\
\text { familiar al } \\
\text { embarazo }\end{array}$ & $\begin{array}{l}\text { Afrontamiento } \\
\text { familiar ineficaz } \\
\text { Alteración de los } \\
\text { procesos } \\
\text { familiares }\end{array}$ & $\begin{array}{l}\text { Que la familia se } \\
\text { adapte a la } \\
\text { situación ce } \\
\text { embarazo }\end{array}$ & $\begin{array}{l}\text { Educación } \\
\text { Apoyo } \\
\text { psicológico }\end{array}$ & $\begin{array}{l}\text { Apoyo educativo } \\
\text { Parcialmente } \\
\text { compensatorio }\end{array}$ & $\begin{array}{l}\text {-Identificar posibles tensiones y fuentes de apoyo } \\
\text { para la familia } \\
\text {-Apoyo en la toma de decisiones } \\
\text {-Indicar el uso de los sistema de apoyo } \\
\text { disponibles } \\
\text {-Referencia a profesional si alteración persiste }\end{array}$ \\
\hline
\end{tabular}

Cuadro I. Planificación de enfermería según requisitos de autocuidado del desarrollo particularizados en embarazadas. 


\begin{tabular}{|c|c|c|c|c|}
\hline $\begin{array}{l}\text { ReQuisito de } \\
\text { autocuidado }\end{array}$ & Método general & Grupos de operaciones requeridas & Factores condicionantes & $\begin{array}{l}\text { Déficit de } \\
\text { Autocuidado }\end{array}$ \\
\hline $\begin{array}{l}\text { Mantener aporte de } \\
\text { lácteos no inferior a } \\
1.000 \mathrm{mg} / 24 \\
\text { horas }^{(14)}\end{array}$ & $\begin{array}{l}\text { Ingestión por boca } \\
\text { de leche, Quesillo, } \\
\text { yogurt u otro } \\
\text { derivado lácteo }\end{array}$ & $\begin{array}{l}\text {-Buscar y validar el conocimiento del requisito } \\
\text {-Preparación de los alimentos } \\
\text {-Consumir los alimentos lácteos medidos y } \\
\text { registrar lo consumido. } \\
\text {-Auto controlar para detectar intolerancia a } \\
\text { estos alimentos } \\
\text {-Comunicar resultados del control al } \\
\text { profesional Que lo ha prescrito }\end{array}$ & $\begin{array}{l}\text {-Disponibilidad y adecuación de } \\
\text { los recursos } \\
\text {-Estado de salud: intolerancia a } \\
\text { los lácteos }\end{array}$ & $\begin{array}{l}\text { Ingestión de lácteos } \\
\text { inferior a } 1.000 \\
\mathrm{mg} / 24 \text { horas }\end{array}$ \\
\hline $\begin{array}{l}\text { Entrenamiento para } \\
\text { la relajación y } \\
\text { fortalecimiento de la } \\
\text { musculatura del piso } \\
\text { pelviano y abdomen } \\
\text { para el parto }\end{array}$ & $\begin{array}{l}\text { Realizar ejercicios } \\
\text { de respiración y } \\
\text { fortalecimiento de la } \\
\text { musculatura del piso } \\
\text { pelviano y abdomen } \\
\text { para el parto }\end{array}$ & $\begin{array}{l}\text {-Buscar y validar el conocimiento del requisito } \\
\text {-Preparación del material y entorno } \\
\text {-Realizar ejercicios de acuerdo a indicaciones } \\
\text {-Autocontrol para detectar efectos, deseados o } \\
\text { adversos } \\
\text {-Comunicar resultados del control al } \\
\text { profesional Que lo ha prescrito }\end{array}$ & -Estilo de vida sedentario, & $\begin{array}{l}\text {-Deficiente uso de } \\
\text { técnicas de } \\
\text { relajación y } \\
\text { fortalecimiento de } \\
\text { la para el parto }\end{array}$ \\
\hline $\begin{array}{l}\text { Monitoreo del } \\
\text { proceso gestacional }\end{array}$ & $\begin{array}{l}\text { Asistir a control } \\
\text { prenatal }\end{array}$ & $\begin{array}{l}\text {-Buscar y validar el conocimiento del requisito } \\
\text {-Preparación del material y recursos } \\
\text {-Asistir a control } \\
\text {-Autocontrol para informar modificaciones } \\
\text { esperadas y su efectos } \\
\text {-Comunicar resultados del autocontrol al } \\
\text { profesional Que la atiende }\end{array}$ & $\begin{array}{l}\text {-Disponibilidad y adecuación de } \\
\text { los recursos } \\
\text {-Factores del sistema de cuidados } \\
\text { de salud (ejm. horario de citas) } \\
\text {-Orientación sociocultural }\end{array}$ & $\begin{array}{l}\text { Proceso de } \\
\text { gestación no } \\
\text { monitorizado }\end{array}$ \\
\hline $\begin{array}{l}\text { Aporte profiláctico } \\
\text { de } 60-100 \mathrm{mg} \text { de } \\
\text { hierro }^{(15)}\end{array}$ & $\begin{array}{l}\text { Ingestión por boca } \\
\text { de preparados Que } \\
\text { aseguren } 60-100 \\
\text { mg de hierro } \\
\text { elemental }\end{array}$ & $\begin{array}{l}\text {-Buscar y validar el conocimiento del requisito } \\
\text {-Preparación del material (cítricos para } \\
\text { favorecer absorción) } \\
\text {-Consumir preparados según indicación } \\
\text {-Autocontrol para detectar intolerancia a } \\
\text { preparados } \\
\text {-Comunicar resultados del control al } \\
\text { profesional Que lo ha prescrito }\end{array}$ & $\begin{array}{l}\text {-Estado de salud: intolerancia a } \\
\text { los preparados }\end{array}$ & $\begin{array}{l}\text { Aporte profiláctico } \\
\text { inferior a } 60-100 \\
\mathrm{mg} \text { de hierro }\end{array}$ \\
\hline $\begin{array}{l}\text { Mantener aporte de } \\
\text { alimentos ricos en } \\
\text { fibra }\end{array}$ & $\begin{array}{l}\text { Ingestión por boca } \\
\text { de alimentos ricos } \\
\text { en fibra }\end{array}$ & $\begin{array}{l}\text {-Buscar y validar el conocimiento del requisito } \\
\text {-Preparación de alimentos } \\
\text {-Consumir los alimentos ricos en fibra y } \\
\text { registrar lo consumido. } \\
\text {-Autocontrol para detectar efectos deseados } \\
\text {-Comunicar resultados del control al } \\
\text { profesional Que monitoriza la gestación }\end{array}$ & $\begin{array}{l}\text {-Estado de salud: intolerancia a } \\
\text { los alimentos }\end{array}$ & Constipación \\
\hline $\begin{array}{l}\text { Adaptación familiar } \\
\text { a la situación de } \\
\text { embarazo }\end{array}$ & $\begin{array}{l}\text { Proceso de } \\
\text { identificación } \\
\text { familiar }\end{array}$ & $\begin{array}{l}\text {-Buscar y validar el conocimiento del requisito } \\
\text { Preparación de materiales y entorno } \\
\text { (preparación de ropa, dormitorio para el recién } \\
\text { nacido) } \\
\text {-Comunicación con (familiares/amigos) para } \\
\text { compartir y elaborar la experiencia. } \\
\text {-Autocontrol para alteraciones del proceso de } \\
\text { adaptación } \\
\text {-Comunicar resultados en el control con } \\
\text { profesional }\end{array}$ & $\begin{array}{l}\text {-Factores del sistema familiar } \\
\text {-Estado de salud }\end{array}$ & $\begin{array}{l}\text { Ineficaz adaptación } \\
\text { familiar al embarazo }\end{array}$ \\
\hline
\end{tabular}

Cuadro 2. Elementos de un sistema de acción para satisfacer requisitos de autocuidado del desarrollo particularizado en embarazadas. 


\begin{tabular}{|c|c|c|c|c|}
\hline $\begin{array}{l}\text { Requisito de } \\
\text { autocuidado }\end{array}$ & Método general & Grupos de operaciones requeridas & Factores condicionantes & Déficit de Autocuidado \\
\hline $\begin{array}{l}\text { Ingestión de calcio } \\
(1500 \mathrm{mg} / \mathrm{d} \text { sin } \\
\text { TRH) y vitamina D } \\
(400 \mathrm{U} / \mathrm{d})^{(16)}\end{array}$ & $\begin{array}{l}\text { Ingestión por boca } \\
\text { de preparados Que } \\
\text { aseguren } \\
\text { requerimientos }\end{array}$ & $\begin{array}{l}\text {-Buscar y validar el conocimiento del requisito } \\
\text { (significado, duración y efectos propuestos) } \\
\text {-Preparación del material } \\
\text {-Consumir preparados según indicación } \\
\text {-Autocontrol para detectar intolerancia a } \\
\text { preparados } \\
\text {-Comunicar resultados del control al } \\
\text { profesional Que lo ha prescrito }\end{array}$ & $\begin{array}{l}\text {-Disponibilidad de los } \\
\text { recursos }\end{array}$ & $\begin{array}{l}\text { Ingestión de calcio y vit. D } \\
\text { inferior a requerimien-tos }\end{array}$ \\
\hline $\begin{array}{l}\text { Fortalecer la } \\
\text { musculatura del piso } \\
\text { pelviano para } \\
\text { favorecer el control } \\
\text { vesical }\end{array}$ & $\begin{array}{l}\text { Realizar ejercicios } \\
\text { de kejel }\end{array}$ & $\begin{array}{l}\text {-Buscar y validar el conocimiento del requisito } \\
\text { - Preparación del material y el entorno } \\
\text {-Realizar ejercicios de acuerdo a indicaciones } \\
\text { diariamente. } \\
\text {-Autocontrol para detectar evidencias de los } \\
\text { efectos deseados } \\
\text {-Comunicar los resultados del control al } \\
\text { profesional Que lo ha prescrito }\end{array}$ & $\begin{array}{l}\text {-Estilo de vida sedentario. } \\
\text {-Orientación sociocultural }\end{array}$ & $\begin{array}{l}\text { Disminución de tono de } \\
\text { musculatura del perineo y } \\
\text { déficit de control vesical }\end{array}$ \\
\hline Ejercicio terapéutico & $\begin{array}{l}\text { Realizar alguna de } \\
\text { las siguientes } \\
\text { actividades por } \\
45 \text { minutos 3veces } \\
\text { /semana: } \\
\text {-ejercicios aeróbicos } \\
\text {-tai-chi,, bailar } \\
\text {-marcha rápida } \\
\end{array}$ & $\begin{array}{l}\text {-Buscar y validar el conocimiento del requisito } \\
\text { - Preparación del material y entorno } \\
\text {-Realizar ejercicio terapéutico de acuerdo a } \\
\text { indicaciones } \\
\text {-Autocontrol para detectar evidencias de los } \\
\text { efectos, deseados o adversos } \\
\text {-Comunicar los resultados del control al } \\
\text { profesional Que lo ha prescrito }\end{array}$ & $\begin{array}{l}\text {-Estilo de vida sedentario. } \\
\text {-Disponibilidad de los } \\
\text { recursos }\end{array}$ & $\begin{array}{l}\text { Déficit de ejercicio } \\
\text { terapéutico }\end{array}$ \\
\hline $\begin{array}{l}\text { Evaluación clínica } \\
\text { anual }^{(17)}\end{array}$ & $\begin{array}{l}\text { Asistir a control } \\
\text { climaterio con } \\
\text { profesional para } \\
\text { evaluación anual }\end{array}$ & $\begin{array}{l}\text { Buscar y validar el conocimiento del requisito } \\
\text {-Preparación del material y recursos } \\
\text {-Autocontrol para detectar } \\
\text { síntomas/manifestaciones esperadas durante } \\
\text { este período y sus efectos } \\
\text {-Comunicar resultados del autocontrol al } \\
\text { profesional que la atiende }\end{array}$ & $\begin{array}{l}\text {-Disponibilidad de los } \\
\text { recursos } \\
\text {-Factores del sistema de } \\
\text { cuidados de salud (ejm. } \\
\text { modalidades de } \\
\text { tratamiento) }\end{array}$ & $\begin{array}{l}\text { Proceso de climaterio no } \\
\text { monitorizado }\end{array}$ \\
\hline $\begin{array}{l}\text { Lubricación mucosa } \\
\text { vaginal previo a la } \\
\text { relación sexual }\end{array}$ & $\begin{array}{l}\text { Aplicar lubricante } \\
\text { en vagina previo a la } \\
\text { relación sexual }\end{array}$ & $\begin{array}{l}\text { Buscar y validar el conocimiento del reQuisito } \\
\text { - Preparación del material } \\
\text {-Aplicación de lubricante en vaginal según } \\
\text { indicación } \\
\text {-Autocontrol para detectar intolerancia a } \\
\text { preparados vaginales } \\
\text {-Comunicar resultados del control al } \\
\text { profesional Que lo ha prescrito }\end{array}$ & $\begin{array}{l}\text {-Orientación sociocultural } \\
\text {-Disponibilidad de los } \\
\text { recursos }\end{array}$ & $\begin{array}{l}\text { Adaptación individual } \\
\text { ineficaz por molestias } \\
\text { producidas durante la } \\
\text { relación sexual }\end{array}$ \\
\hline
\end{tabular}

Cuadro 3. Elementos de un sistema de acción para satisfacer requisitos de autocuidado del desarrollo particularizado en mujeres en etapa de climaterio. 


\begin{tabular}{|c|c|c|c|c|c|}
\hline $\begin{array}{l}\text { Déficit de } \\
\text { Autocuidado }\end{array}$ & $\begin{array}{l}\text { Diagnóstico de } \\
\text { enfermería }\end{array}$ & Objetivo & Método de ayuda & Tipo de sistema & Intervenciones de Enfermería \\
\hline $\begin{array}{l}\text { Ingestión de } \\
\text { calcio y vitamina } \\
\text { D inferior a lo } \\
\text { recomendado }\end{array}$ & $\begin{array}{l}\text { Alteración de la } \\
\text { nutrición: } \\
\text { consumo inferior } \\
\text { de los } \\
\text { requerimientos } \\
\text { de calcio y } \\
\text { vitamina D }\end{array}$ & $\begin{array}{l}\text { Que la mujer } \\
\text { adopte medidas } \\
\text { para favorecer la } \\
\text { ingesta de calcio } \\
\text { y vitamina D }\end{array}$ & Educación & Apoyo educativo & $\begin{array}{l}\text {-Proporcionar instrucción acerca de necesidades } \\
\text { de nutrientes durante la etapa de climaterio } \\
\text {-Proporcionar instrucción acerca de: medidas } \\
\text { Que favorezcan la absorción de calcio y vitamina } \\
\text { D y, disponibilidad de costearlos. }\end{array}$ \\
\hline $\begin{array}{l}\text { Disminución de } \\
\text { tono de } \\
\text { musculatura del } \\
\text { perineo y déficit } \\
\text { de control vesical }\end{array}$ & $\begin{array}{l}\text { Déficit de control } \\
\text { vesical }\end{array}$ & $\begin{array}{l}\text { Que la mujer } \\
\text { adopte medidas } \\
\text { para favorecer el } \\
\text { fortalecimiento } \\
\text { de la musculatura } \\
\text { del perineo y } \\
\text { control vesical }\end{array}$ & Guiar y dirigir & $\begin{array}{l}\text { Apoyo educativo } \\
\text { Parcialmente } \\
\text { compensatorio }\end{array}$ & $\begin{array}{l}\text {-Proporcionar instrucción acerca de } \\
\text { modificaciones anatomo-fisiológicas Que se } \\
\text { producen durante el período de climaterio a } \\
\text { causa del déficit estrogénico } \\
\text {-Indicación de ejercicios Que fortalezcan } \\
\text { musculatura del perineo } \\
\text {-Referencia a control profesional }\end{array}$ \\
\hline $\begin{array}{l}\text { Déficit de } \\
\text { ejercicio } \\
\text { terapéutico }\end{array}$ & $\begin{array}{l}\text { Déficit de } \\
\text { actividad física }\end{array}$ & $\begin{array}{l}\text { Que la mujer } \\
\text { realice ejercicio } \\
\text { terapéutico } \\
\text { según } \\
\text { indicaciones }\end{array}$ & $\begin{array}{l}\text { Educación } \\
\text { Guiar y dirigir }\end{array}$ & $\begin{array}{l}\text { Apoyo educativo } \\
\text { Parcialmente } \\
\text { compensatorio }\end{array}$ & $\begin{array}{l}\text {-Proporcionar instrucción acerca de } \\
\text { modificaciones anatomo-fisiológicas Que se } \\
\text { producen durante Que se producen durante el } \\
\text { período de climaterio } \\
\text {-Indicación de ejercicio terapéutico previa } \\
\text { evaluación profesional } \\
\text { - Referencia a control profesional }\end{array}$ \\
\hline $\begin{array}{l}\text { Proceso de } \\
\text { climaterio no } \\
\text { monitorizado }\end{array}$ & $\begin{array}{l}\text { Mantenimiento } \\
\text { de la salud } \\
\text { alterado }\end{array}$ & $\begin{array}{l}\text { Que la mujer } \\
\text { mantenga un } \\
\text { monitoreo anual } \\
\text { del proceso de } \\
\text { climaterio }\end{array}$ & $\begin{array}{l}\text { Educación } \\
\text { Guiar y dirigir }\end{array}$ & $\begin{array}{l}\text { Apoyo educativo } \\
\text { Parcialmente } \\
\text { compensatorio }\end{array}$ & $\begin{array}{l}\text {-Proporcionar instrucción acerca de objetivos del } \\
\text { control de climaterio } \\
\text {-Realizar control climaterio } \\
\text {-Referencia a control con otros profesionales } \\
\text {-Solicitud exámenes de rutina }\end{array}$ \\
\hline $\begin{array}{l}\text { Lubricación } \\
\text { mucosa vaginal }\end{array}$ & $\begin{array}{l}\text { Adaptación } \\
\text { individual } \\
\text { ineficaz por } \\
\text { molestias } \\
\text { producidas } \\
\text { durante la } \\
\text { relación sexual }\end{array}$ & $\begin{array}{l}\text { Que la mujer } \\
\text { adopte medidas } \\
\text { para favorecer la } \\
\text { lubricación } \\
\text { vaginal previo a } \\
\text { la relación sexual }\end{array}$ & $\begin{array}{l}\text { Educación } \\
\text { Guiar y dirigir }\end{array}$ & $\begin{array}{l}\text { Apoyo educativo } \\
\text { Parcialmente } \\
\text { compensatorio }\end{array}$ & $\begin{array}{l}\text {-Proporcionar instrucción acerca de } \\
\text { modificaciones anatomo-fisiológicas Que se } \\
\text { producen durante el período de climaterio a } \\
\text { causa del déficit estrogénico } \\
\text { - Indicación de uso de lubricante soluble en agua } \\
\text {-Referencia a control profesional }\end{array}$ \\
\hline
\end{tabular}

Cuadro 4. Planificación de enfermería según reQuisitos de autocuidado del desarrollo particularizados en mujeres en etapa de climaterio. 